\section{Experiencia de enseñanza-}

\section{aprendizaje con aula virtual en}

\section{el acompañamiento pedagógico}

Teaching and learning experience

\section{with a virtual classroom in the}

field of pedagogical support

due to Covid-19

Leda Beatriz Digión* | Margarita María Álvarez**

Recepción del artículo: 23/9/2020 | Aceptación para publicación: 14/1/2021 | Publicación: 26/3/2021

\section{RESUMEN}

En este trabajo se describe una propuesta pedagógica de dictado de una asignatura de grado, que tenía originalmente modalidad presencial, y fue sostenida con la modalidad e-learning en el ciclo lectivo 2020 debido a la situación socio sanitaria ocasionada por el Covid-19. Se aplicó un marco analítico basado en el modelo pedagógico de dimensiones, adaptado para el diseño virtual de la asignatura Informática Médica, de la carrera de Medicina de la Universidad Nacional de Santiago del Estero, Argentina. El dimensionamiento propuesto en el ámbito del proceso de acompañamiento pedagógico proyectado por las autoridades de la facultad permitió programar la participación activa de los estudiantes y organizar el seguimiento del aprendizaje, a través de los ejes propuestos por la cátedra: trabajos individuales, trabajos grupales, interacciones e intervenciones. En cuanto a los resultados obtenidos sobre la mediación educativa en el aula virtual, se identificaron y propusieron las pautas y los recursos en una nueva dimensión pedagógica sobre estrategias didácticas. Así, la dimensión propuesta se considera un componente altamente aprovechable para el trabajo docente en la búsqueda de un estudiante autónomo respecto al logro de metas en su proceso de enseñanza-aprendizaje, como lo muestra la experiencia realizada.

\section{Abstract}

This work describes a pedagogical proposal for the dictation of a degree course, which originally had a face-to-face modality, sustained with the e-learning modality in the 2020 school year due to the social health situation caused by Covid-19. An analytical framework based on the pedagogical model of dimensions was applied, adapted for the virtual design of the Medical Informatics subject, of the Medicine career of the National University of Santiago del Estero, Argentina. The proposed dimensioning in the field of the pedagogical accompaniment process projected by the authorities of the faculty, allowed to program the active participation of the students and organize the monitoring of learning, through the axes proposed by the chair: individual work, group work, interactions and interventions. Regarding the results obtained on educational mediation in the virtual classroom, it was possible to identify and propose the guidelines and resources in a new pedagogical dimension on didactic strategies; thus, this proposed dimension is considered a highly usable component for teaching work in search of an autonomous student, in terms of achieving goals in their teaching and learning process, as shown by the presented experience. 


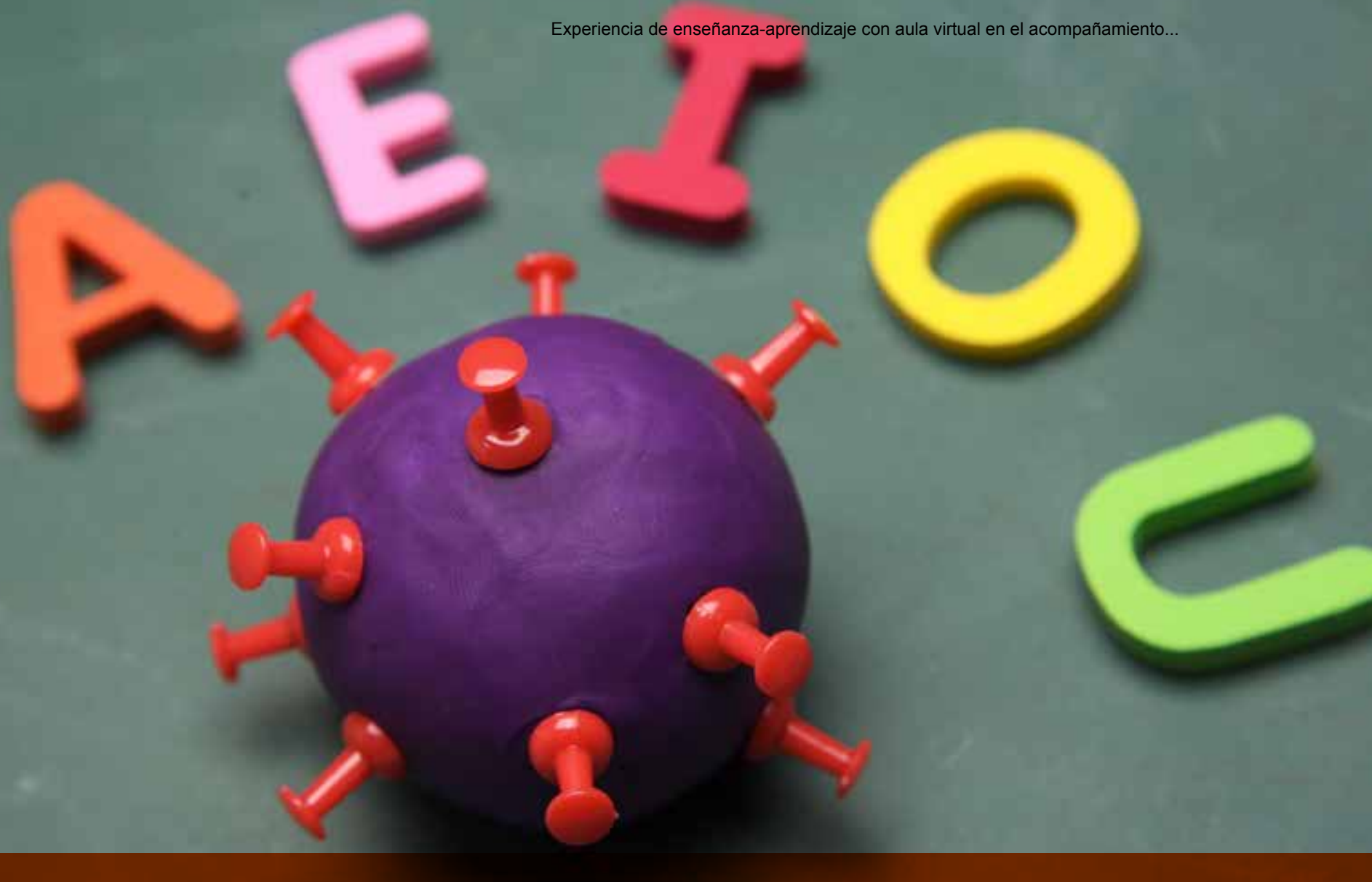

\section{INTRODUCCIÓN}

T a dinámica con la que se desarrolla la sociedad 1 actual, producto de la pandemia de Covid-19, impacta fuertemente en la esfera de la educación. Ante esta situación, se ha tenido la necesidad de explorar modelos pedagógicos y tecnológicos que permitan adaptar la educación presencial para concretarla en espacios virtuales. Debido a esto, en la carrera de Medicina dictada en la Facultad de Ciencias Médicas, dependiente de la Universidad Nacional de Santiago del Estero, Argentina, con una modalidad presencial de carácter permanente, se estableció el dictado de las asignaturas en forma virtual como acompañamiento pedagógico.

En estas condiciones, los docentes se han visto en la necesidad de incorporar o reformular las aulas virtuales (AV), al considerarlas como el único espacio de enseñanza y de aprendizaje que tienen los estudiantes para cumplir con los objetivos de cada asignatura. Por ello, en este documento se presenta una propuesta pedagógica para el curso de Informática Médica (IM) dentro del ámbito del acompañamiento pedagógico requerido por la facultad.

Para el diseño y construcción de las AV de IM se consideraron las siguientes cuestiones: ¿dónde se enseña (contexto)?, ¿qué se enseña (contenidos, problemas, competencias)?, ¿̇para qué se enseña (finalidades educativas)?, ¿qué tipos de interacciones y de mediaciones se dan entre el sujeto que enseña y el sujeto que aprende (concepciones de sujeto, interacción y mediación)? y ccómo se verifica la enseñanza y el aprendizaje (evaluación)? (Rodríguez, 2006). A fin de complementar este enfoque, se 
tomaron en cuenta las dimensiones especificadas por Area y Adell (2009) para el diseño de AV, lo que proporcionó la oportunidad de revisar el contenido de instrucción, las experiencias planificadas y las situaciones evaluativas antes de su implementación.

El seguimiento y el control de la gestión de los estudiantes en la modalidad virtual son unas de las principales actividades en las que los docentes y los alumnos se complementan unos a otros. Este proceso se realizó en un marco conceptual adaptado de las dimensiones usadas para la construcción de $\mathrm{AV}$, donde se definieron los ejes correspondientes. Esto permitió tomar acciones proactivas para el grupo actual y que, a su vez, pudieran beneficiar académicamente al alumnado del curso siguiente.

La visión que se propone en este trabajo, en relación con el uso y el aprovechamiento de las AV, muestra un modelo instructivo que puede utilizarse en cursos virtuales, incorpora el acompañamiento pedagógico y el seguimiento del estudiante con actividades sumativas. A su vez, se busca que este modelo signifique un aporte de propuestas de mejora al curso analizado, hasta su formalización con evaluaciones para el cierre académico.

En las siguientes secciones se presenta la conceptualización de las AV y una revisión de trabajos relacionados con sus dimensiones, la metodología utilizada, donde se incluye una revisión del modelo pedagógico del AV basado en estas dimensiones, la descripción de la propuesta pedagógica para las AV de la asignatura Informática Médica, los resultados de los aprendizajes y, por último, las conclusiones y los potenciales trabajos futuros.

\section{ESPACIOS VIRTUALES DE APRENDIZAJE}

Conceptualmente, se entienden a los entornos virtuales como el recurso web o software con finalidades educativas que permite gestionar todas las actividades características de la enseñanza y el aprendizaje de una asignatura o curso. Estos facilitan el anclaje de los contenidos de aprendizaje en diversos y enriquecedores formatos digitales, además posibilitan las comunicaciones verticales y horizontales, tanto síncronas como asíncronas, el trabajo colaborativo, el control y el seguimiento de todos los usuarios, así como la gestión y la administración del proceso (García Aretio, 2014, citado en García Arieto, 2020). En resumen, se trata de una estructura tecnológica que responde a las exigencias de un determinado diseño pedagógico perfectamente integrado.

Estos entornos o plataformas virtuales de aprendizaje encajan por completo en nuestra concepción de educación a distancia como diálogo didáctico mediado, es decir, finalidades educativas que se concretan en aprendizajes valiosos (componente pedagógico), diálogos, comunicación o interacción necesarias (componente social) y mediación a través del software correspondiente (componente tecnológico) (García Aretio, 2014, citado por García Arieto, 2020).

Un buen exponente de los primeros pasos del e-learning fueron las plataformas o Learning Management Systems (LMS), que permitían anclar contenidos interactivos y facilitar recursos digitales para la comunicación síncrona y asíncrona con imagen y sonido. Posteriormente, estas plataformas se abrieron a las posibilidades de la web 2.o y las redes sociales (García Peñalvo y Seoane Pardo, 2015).

Un LMS es una herramienta de software que permite gestionar contenidos, actividades y usuarios; en cambio, las AV integran la metodología y la estructura a partir de las cuales se disponen los recursos con fines de aprendizaje (Humanante Ramos, 2016, citado en Humanante-Ramos, Fernández-Acevedo y Jiménez, 2019). Dentro de los LMS existen diversas plataformas, tanto de licencia comercial como de libre acceso; de estas últimas, destaca la plataforma Moodle (Modular ObjectOriented Dynamic Learning), como un sistema integrado, robusto y seguro para crear ambientes de aprendizaje personalizados. 
aprendizaje reconfigurable, parecido a un sistema de bloques de construcción estilo Lego que permita habilitar una experiencia de enseñanza-aprendizaje en todo el proceso educativo (Brown, Dehoney y Milichap, 2015, citado en TEDU, 2016, p. 10).

Se define al AV como un espacio o entorno creado virtualmente con la intencionalidad de que un estudiante obtenga experiencias de aprendizaje mediante recursos y materiales formativos, bajo la supervisión e interacción con un profesor. A través de ese entorno el alumno puede acceder y desarrollar una serie de acciones similares a las que acontecen en un proceso de enseñanza presencial, como conversar, leer documentos, realizar ejercicios, formular preguntas al docente, trabajar en equipo, entre otras.

Este espacio de encuentro educativo no es azaroso, sino intencional, regulado, planificado y dirigido por el docente. Esto implica que el estudiante, al acceder a una $\mathrm{AV}$, debe obtener experiencias o vivencias de situaciones potenciales de aprendizaje de forma similar a lo que ocurriría en los escenarios presenciales (Area y Adell, 2009). Por ello, las plataformas virtuales deben

\section{Se define al aula virtual}

como un espacio o entorno creado virtualmente con

la intencionalidad de que un estudiante obtenga experiencias de aprendizaje mediante recursos y materiales formativos, bajo la supervisión e interacción con un profesor sustentarse en un modelo pedagógico que articule el uso de metodologías activas de enseñanza, aprendizaje y evaluación; adicionalmente, también resulta necesario una fuerte inversión en equipos docentes y recursos tecnológicos (Hoyos Giraldo, 2015).

Las tecnologías de la información y la comunicación (TIC) y las plataformas virtuales, como apoyo o alternativa para la educación presencial, parten de premisas optimistas en torno a que estas logran estímulos auditivos y visuales que propician el aprendizaje y la reconstrucción del conocimiento, lo que permite desarrollar nuevas experiencias docentes, así como habilidades y destrezas en los docentes y los estudiantes (Regueyra, 2011, citado en Granados-Zúñiga, 2019).

\section{ANTECEDENTES}

Existen numerosos antecedentes sobre las dimensiones de las AV; con el fin de evaluarlas o diseñarlas, a continuación se presentan algunas de las propuestas revisadas para el marco analítico de nuestro modelo pedagógico. Los autores Barberá y Badia (2005) plantearon dos grandes dimensiones de las AV: por un lado, las relativas al diseño y la planificación (objetivos, materiales, agrupamientos, etcétera) y, por otro, aquellas relativas a su implementación y desarrollo (fases y rasgos de cada fase).

Khan (2005) propone ocho dimensiones que deben considerarse en los cursos en línea: institucional, de gestión, tecnológica, pedagógica, ética, diseño de la interfaz, apoyos y evaluación. Las más significativas son: a) la pedagógica, que incluye los objetivos, contenidos, organización, metodología, estrategia didáctica, etcétera; b) la tecnológica, que se refiere a los elementos tecnológicos que soportan los cursos en línea; c) la de diseño de la interfaz, que incluye cómo se ve, cómo se siente y cómo responde la interfaz del curso; d) la de evaluación, que considera la evaluación de los aprendizajes; y e) la de orientación 
en línea, la función del docente en la atención, seguimiento y retroalimentación de los avances en el proceso de aprendizaje de sus estudiantes.

De forma similar, Area y Adell (2009) identificaron en un AV cuatro grandes dimensiones pedagógicas: la informativa, la experimental o praxis, la tutorial y evaluativa, y la comunicativa. Por su lado, en el trabajo presentado por García Fernández (2014) se estudia la actividad de los docentes y los estudiantes en las AV para tipificar su uso, con la intención de orientar el perfeccionamiento de la formación de profesionales de la educación. El autor considera dos dimensiones: de contenido, que está formado por todos los textos digitales que aporten información valiosa para el aprendizaje de los estudiantes, y de actividad, que incluye atención grupal e individual, pruebas y exámenes, así como el uso de las herramientas de trabajo.

Posteriormente, Monteza Calderón (2016) buscó diagnosticar, caracterizar y comprender el uso de las TIC y las AV por los profesores y estudiantes de la Facultad de Humanidades de la Universidad Católica Santo Toribio de Mogrovejo (USAT), en Perú. Para ello, se definieron las dimensiones uso, dominio y utilidad de las TIC, y las dimensiones informativa, comunicativa, práctica y tutorial de las AV. Años más tarde, Area (2018) consideró dos enfoques pedagógicos para la creación y el uso de AV: el enfoque basado en el paradigma de la pedagogía expositiva y el basado en el paradigma de la pedagogía activa o experiencial.

El primero se caracteriza por concebir los entornos en línea como espacios de presentación del conocimiento para el aprendizaje por recepción, así como para el desarrollo de procesos de enseñanza-aprendizaje individuales y estandarizados para todos. En el segundo enfoque, de pedagogía activa o experiencial, se conciben como espacios organizados en función de la demanda de cumplimentación de actividades -tareas y proyectos que el alumnado tiene que desarrollar en interacción con otros estudiantes, empleando distintos

\section{Area (2018) consideró dos}

\section{enfoques pedagógicos para}

la creación y el uso de AV: el

enfoque basado en el paradigma

de la pedagogía expositiva y el

basado en el paradigma de la

pedagogía activa o experiencial

objetos y recursos en línea-, y de la flexibilidad y adaptabilidad a los ritmos y necesidades personales de los estudiantes.

\section{METODOLOGÍA}

Para realizar la propuesta pedagógica de las AV de IM, y el seguimiento de los aprendizajes de los estudiantes, en primer lugar, se definieron las dimensiones pedagógicas a considerar, posteriormente se diseñaron los instrumentos informativos, de evaluación y de seguimiento, y se realizó un análisis de los resultados obtenidos.

$\mathrm{El}$ diseño de la propuesta pedagógica para el $\mathrm{AV}$ de IM que se presenta se fundamentó en el modelo pedagógico del aula virtual basado en las dimensiones desarrolladas por Area y Adell (2009):

- Dimensión informativa: entendida como el "conjunto de recursos, materiales o elementos que presentan información o contenido diverso para el estudio autónomo por parte del alumnado" (p. 8). Se refieren a los diferentes recursos informativos que ayudan a los estudiantes a comprender los contenidos de la asignatura. Esta dimensión abarca un conjunto de materiales de distinta naturaleza -de elaboración propia o ajena-, que desde el aula 
permiten a los alumnos acceder a los conocimientos (Porro, 2017).

- Dimensión experimental: plantea actividades individuales y grupales con el fin de desarrollar experiencias activas de aprendizaje en la construcción del conocimiento.

- Dimensión evaluativa: refiere a las evaluaciones, de forma que se descubra el avance del aprendizaje de los estudiantes y se acredite la materia. Los autores consideran en esta dimensión las tutorías.

- Dimensión comunicativa: refiere al conjunto de recursos y acciones de interacción social entre los estudiantes y el profesor.

Tras considerar estas dimensiones, se propuso complementarlas con la dimensión mediación educativa en el $\mathrm{AV}$, donde se establecen las estrategias didácticas y los recursos necesarios para la implementación de AV, para lograr la consecución de los objetivos de aprendizaje y cumplimentar con las dimensiones anteriores de manera planificada.

\section{PROPUESTA PEDAGÓGICA PARA EL AULA VIRTUAL DE INFORMÁTICA MÉDICA}

\section{Estudio de la asignatura y el perfil del alumno}

Con el propósito de diseñar la propuesta, se consideró el contexto, los contenidos, la modalidad y las finalidades educativas de la asignatura IM, cuyo objetivo general es conocer y utilizar reflexivamente las herramientas de la informática en el campo de la medicina, como recurso para la práctica y el desarrollo profesional. Desde su comienzo en 2017 , la modalidad de la asignatura fue presencial; en 2018 se implementó el AV bajo la plataforma Moodle como soporte a la presencialidad, lo que posibilitó el acceso a los materiales educativos y al soporte tecnológico, además de la comunicación con los estudiantes y generar un espacio de actividades.
Como consecuencia del aislamiento social, preventivo y obligatorio decretado a raíz del Covid-19, en 2020 el AV ha tenido una nueva configuración funcional, debido al continuo proceso de refinamiento y adaptación por la nueva modalidad. El AV fue planteada como única herramienta y soporte, $y$ como principal estrategia educativa para la enseñanza y el aprendizaje de la asignatura; asimismo, se cuidó que cuando el estudiante acceda a un AV obtenga experiencias o vivencias de situaciones potenciales de aprendizaje significativo de forma similar a como lo haría en los escenarios presenciales, leer textos, formular preguntas, resolver problemas, entregar sus trabajos, participar en un debate o rendir un examen, entre otras tareas académicas. Para lograr esto, en el diseño y manejo de las AV de IM se trabajaron los diferentes aspectos que conforman las dimensiones consideradas.

\section{Dimensión informativa}

En el AV de la asignatura se han incluido tres tipos de información: general, específica sobre la asignatura y las pautas metodológicas. En estas se detalla información de la materia, como la bienvenida al $\mathrm{AV}$, los integrantes del equipo docente, el programa de la asignatura, sus objetivos, la bibliografía y las condiciones de aprobación.

Debido a que el AV fue organizada a partir de los núcleos temáticos descritos en la planificación de la materia, en cada unidad se ha incluido información específica de la asignatura, elaborada por los docentes de la cátedra, tanto de la teoría como de la práctica. Este material se acompaña de otros recursos de información multimediales de autores externos -en general se han incluido textos obligatorios y complementarios, así como enlaces a videos y softwares específicos de la temática de la unidad.

En el repositorio también se agregó información sobre pautas y referencias metodológicas para formalizar la presentación y el desarrollo de los trabajos de los estudiantes, así como de otros resultados obtenidos en las actividades colaborativas del AV. 


\section{Dimensión experimental}

En esta dimensión se plantean actividades grupales tanto de teoría como de práctica. El trabajo grupal de teoría se basó en generar un proceso reflexivo inicial de mejora y desarrollo continuo, respecto a los materiales de estudio, las interacciones producidas y la plataforma tecnológica. Asimismo, la asignatura propuso la elaboración de seis trabajos prácticos de forma grupal sobre los siguientes temas: búsqueda de la información médica, Excel, Word, EpiInfo, digitalización de imágenes y editor de presentaciones e Historias Clínicas.

En la tabla 1 se presentan los objetivos y los resultados de aprendizaje esperados de los dos trabajos prácticos más representativos. En todos se consideraron como criterios principales para el seguimiento de los estudiantes: la entrega del documento en tiempo y forma, su completitud, y la calidad del trabajo presentado.

Debido a que en la actualidad los problemas no pueden ser vistos de manera fragmentaria y descontextualizada, sino que deben ser planteados en un contexto planetario, integrando los saberes para solucionarlos (Nivela Cornejo et al., 2019), se ha propuesto un trabajo grupal de integración horizontal con la asignatura Salud Pública II del mismo semestre. Este tipo de trabajos o proyectos integradores reúnen los conocimientos de varias materias y logran que el estudiante obtenga una competencia completa, y resuelva una problemática en específico. Con estos se busca desarrollar en el estudiante un enfoque interdisciplinario y transdisciplinarios para tratar problemas de la disciplina (Nivela Cornejo et al., 2019).

El trabajo integrador asignado tuvo como objetivo general integrar conocimientos y herramientas trabajados en las materias de Salud Pública II e IM, aplicados en el análisis de la situación de salud de la población, bajo la coordinación de las Unidades Primarias de Atención (UPA) asignada. Con esto, los resultados de aprendizaje desde la IM que se esperan alcanzar son: organizar y manipular los datos recogidos en las UPAs, obtener estadísticas significativas, valorar las tecnologías informáticas para la organización y manipulación de información médica, integrar los saberes relacionados con las disciplinas de estudio, trabajar en forma efectiva como integrante en un trabajo de grupo en ambientes multidisciplinarios, resumir la

Tabla 1. Objetivos y resultados de aprendizaje

\begin{tabular}{|c|c|c|}
\hline $\begin{array}{l}\text { TEMA DE TRABAJOS } \\
\text { PRÁctICOS }\end{array}$ & OBJetivos de APRENDIZAJE & RESULTADOS DE APRENDIZAJE ESPERADOS \\
\hline $\begin{array}{l}\text { Búsqueda de } \\
\text { información } \\
\text { médica }\end{array}$ & $\begin{array}{l}\text { Conocer las estrategias formales } \\
\text { de búsqueda y recuperación de la } \\
\text { información } \\
\text { - Desarrollar la habilidad para realizar } \\
\text { búsquedas adecuadas de información } \\
\text { médica disponibles en internet }\end{array}$ & $\begin{array}{l}\text { - Analizar, seleccionar y aplicar las estrategias } \\
\text { de búsqueda } \\
\text { - Realizar búsquedas en DeCs, en la Biblioteca } \\
\text { Virtual en Salud, y en otros recursos de } \\
\text { información médica disponibles en internet }\end{array}$ \\
\hline $\begin{array}{l}\text { Historia clínica } \\
(\mathrm{HC})\end{array}$ & $\begin{array}{l}\text { Familiarizarse con los Sistemas de } \\
\text { Información (SI) Hospitalaria, en especial } \\
\text { con los sistemas de gestión de } \mathrm{HC} \text {, de tal } \\
\text { forma que permita al estudiante obtener } \\
\text { más conocimientos sobre este tipo de } \\
\text { sistemas }\end{array}$ & $\begin{array}{l}\text { - Enumerar y describir los elementos que } \\
\text { componen un SI de HC } \\
\text { - Comprender las ventajas que conlleva la } \\
\text { utilización de HC electrónicas } \\
\text { - Analizar y categorizar los datos clínicos, y } \\
\text { emplear los softwares de historias clínicas } \\
\text { para su registro y procesamiento } \\
\text { - Instalar los softwares }\end{array}$ \\
\hline
\end{tabular}

Fuente: elaboración propia. 


\section{En la planificación del} trabajo individual de teoría se propuso ofrecer una

\section{estrategia para que el estudiante ayude a desarrollar} su propio aprendizaje, que aplique un instrumento para recoger evidencias

investigación realizada y concluir con una presentación del trabajo.

\section{Dimensión evaluativa}

En las AV se han planteado trabajos de valoración práctica y teórica sobre los temas vistos en la asignatura. En el trabajo individual se realizó una evaluación práctica, consistente en la resolución de problemas mediante el uso de los softwares vistos en la asignatura; mientras que en la planificación del trabajo individual de teoría se propuso ofrecer una estrategia para que el estudiante ayude a desarrollar su propio aprendizaje, que aplique un instrumento para recoger evidencias de este, como requisito del proceso de acompañamiento pedagógico actual, e incentive al aprendizaje en modalidad virtual.

\section{Dimensión comunicativa}

Para la propuesta, en la dimensión comunicativa se han considerado dos categorías: las interacciones y las intervenciones. Las primeras han tomado en cuenta la relación recíproca entre los estudiantes, entre el estudiante y el docente, y entre el estudiante y el AV.
- Interacción alumno-alumno: se concretó en los trabajos teóricos y prácticos grupales. $\mathrm{Al}$ comienzo de la asignatura se organizaron los grupos de estudiantes, estos fijaron el criterio de pertenencia a sus respectivas comisiones. Así, los grupos se conformaron con pares de la misma comisión y permanecieron en el grupo hasta el final de las actividades, salvo algunas excepciones. Además, para el trabajo integrador con Salud Pública II se formaron los grupos de acuerdo al trabajo de las UPAs asignadas.

- Interacción/relación alumnos con profesores: se dio mediante la comunicación sincrónica y asincrónica través de chats, videoconferencias $\mathrm{y}$ foros de consultas para cada tema.

- Interacción estudiante-aula virtual: se tomó como categoría debido a que el AV se consideró como el único espacio de soporte para la enseñanza y el aprendizaje. Tener en cuenta esta relación permite dar seguimiento a los aprendizajes de los estudiantes y detectar a quienes tuvieron problemas técnicos de comunicación o falta de interés en la asignatura.

En el caso de las intervenciones, se han dividido en dos: de los docentes y de los alumnos. El proceso de intervención docente es otro elemento del entorno virtual de aprendizaje, además de los docentes como tutores y los estudiantes como aprendices. En esta instancia de cursado actual, se pueden observar las distintas intervenciones que requieren los estudiantes por parte de los profesores en los espacios de comunicación y en el desarrollo de las actividades; en estas se identificaron los propósitos de información, de organización, de feedback por parte del docente, de respuesta a solicitudes de ayuda, entre otros.

Con el fin de manejar los tiempos establecidos en el curso, además de ordenar y conducir su desarrollo, los docentes concretaron intervenciones de información o contenido. Las fechas de comienzo de cada tema teórico y práctico, los materiales a consultar, el software a instalar, y toda 
la información referente al curso fueron publicados y enviados por los docentes a los alumnos, en numerosos mensajes a través del foro y por correo electrónico. Como docente-tutor, los profesores también realizan intervenciones para ayudar a organizar, apoyar y orientar las tareas de los alumnos, a ordenar los tiempos para su ejecución, o en las secuencias más interesantes en función de las características o estilos de aprendizaje de cada alumno.

En las intervenciones de feedback se realiza una retroalimentación a los estudiantes como una instancia de promoción y construcción de conocimiento y aprendizaje. Esta retroalimentación casi siempre se ofrece en relación con un producto elaborado por los alumnos, es decir, una tarea. En esta intervención se presenta un juicio valorativo acerca de la tarea realizada por el estudiante, de quien, posteriormente, se espera que presente nueva información enlazada con la anterior. Estas intervenciones se pueden observar sobre todo en las devoluciones realizadas a los trabajos prácticos, en la actividad de valoración práctica y en el trabajo de investigación grupal. Sobre las intervenciones para responder a solicitudes específicas de los alumnos, se proporcionó información en respuesta a una demanda planteada por algún estudiante, a través del correo electrónico.

En relación con las intervenciones concretadas por los alumnos, se identificaron los si- guientes propósitos: responder a las demandas planteadas por el tutor -a través de correos electrónicos, son realizadas por los alumnos para cumplimentar con las solicitudes planteadas por el tutor como tareas del curso-, y solicitar ayuda para esclarecer algún contenido conceptual o procedimental -en este punto también se puede esperar la ayuda colaborativa de los compañeros, ya que el foro es el lugar idóneo para este desarrollo-. Ambas intervenciones se reflejan en los correos que los estudiantes envían, en su mayoría de forma privada, a los docentes tutores.

$\mathrm{Al}$ considerar lo anterior, se puede observar en las interacciones e intervenciones los puntos de vista e interpretaciones entre los estudiantes, así como la participación como tutores. No obstante, el protagonismo en el aula lo tienen los estudiantes, pues desempeñan un rol activo en su propio aprendizaje.

\section{Dimensión mediación educativa en el aula virtual}

Las estrategias didácticas, con sus respectiva técnicas y recursos, como la nueva dimensión incorporada, se describe en la tabla 2. Se espera que, a partir de esta información, se proporcione una aplicación centrada en un estudiante autónomo, en cuanto al logro de sus metas en el proceso de enseñanza-aprendizaje.

Tabla 2. Mediación educativa en el aula virtual

\begin{tabular}{|c|c|}
\hline ESTRATEGIAS DIDÁ & Recursos \\
\hline $\begin{array}{l}\text { Para cada unidad de estudio se subió al AV la presentación teórica y práctica preparada } \\
\text { por los docentes, el material bibliográfico y las consignas de actividades de investigación/ } \\
\text { trabajos prácticos. Las estrategias principales empleadas fueron: } \\
\text { - Estudio individual: el alumno realizó el estudio de la unidad y, posteriormente, se le } \\
\text { habilitó en el horario de las clases teóricas un chat o videoconferencia de consultas y } \\
\text { un foro para las consultas individuales } \\
\text { - Tareas individuales/grupales: para resolver la práctica se programaron chats de con- } \\
\text { sultas y videoconferencias en los que se brindaron las explicaciones generales y se } \\
\text { solventaron dudas sobre los ejercicios propuestos } \\
\text { Colaborativas: se realizaron actividades de investigación y desarrollo sobre algunos } \\
\text { temas de la asignatura, con intercambio y fijación de objetivos de estudio }\end{array}$ & $\begin{array}{l}\text { - Presentaciones con } \\
\text { diapositivas } \\
\text { - Videos } \\
\text { - Cuestionarios } \\
\text { - Foros de consulta } \\
\text { - Chats } \\
\text { - Videoconferencia } \\
\text { - Bibliografía seleccio- } \\
\text { nada por la cátedra } \\
\text { - Software específico }\end{array}$ \\
\hline
\end{tabular}




\begin{tabular}{|l|l|}
\hline \multicolumn{1}{|c|}{ ESTRATEGIAS DIDÁCtICAS } & \multicolumn{1}{c|}{ ReCURSOS } \\
\hline $\begin{array}{l}\text { Aprendizaje basado en problemas: se refiere al trabajo grupal que se realiza con la } \\
\text { asignatura Salud Pública II }\end{array}$ & $\begin{array}{l}\cdot \text { Los descritos } \\
\text { anteriormente }\end{array}$ \\
\hline $\begin{array}{l}\text { Actividad de valoración práctica: para el seguimiento de los estudiantes se ha desarrollado } \\
\text { una actividad práctica individual }\end{array}$ & $\begin{array}{l}\text { C Cuestionario } \\
\text { Chat de consulta }\end{array}$ \\
\hline $\begin{array}{l}\text { Cuestionario para autoaprendizaje: se aplicó este instrumento para fijación y recolección } \\
\text { de evidencias de aprendizaje }\end{array}$ & $\begin{array}{l}\text { C Cuestionario } \\
\text { Chat de consulta }\end{array}$ \\
\hline
\end{tabular}

Fuente: elaboración propia.

\section{RESULTADOS DE LOS APRENDIZAJES}

Con base en las dimensiones del modelo pedagógico presentado se han considerado los siguientes ejes procesuales para el seguimiento de los estudiantes:

- Eje 1. Trabajos grupales de práctica y de teoría

- Eje 2. Trabajos individuales de valoración práctica y teórica

- Eje 3. Interacciones

- Eje 4. Intervenciones

La asignatura se puede promocionar con calificación de siete o superior en todas las actividades de aprendizaje, y alcanza a regularizarse con una calificación de cinco o mayor. Las actividades sumativas de valoración y fijación de aprendizaje sirvieron como acciones mediadoras y reflexivas entre el docente y el estudiante, y fueron utilizadas para orientar el acercamiento paulatino y sistemático del grupo de estudiantes. A continuación, se describen los resultados de aprendizaje obtenidos por cada eje.

\section{Eje 1. Trabajos grupales}

Los trabajos grupales se llevaron a cabo a lo largo de la materia, y los resultados obtenidos evidencian un alto rendimiento en los estudiantes en el trabajo de búsqueda de la información médica, con lo que se concluye que los alumnos son capaces de localizar y explotar las principales fuentes de información médica mediante la aplicación de las estrategias pertinentes. En la gráfica 1 se puede observar que, sobre un grupo de 138 alumnos,
17.39\% alcanzó la nota máxima, 3.62 \% la nota de 70 puntos y $77.53 \%$ obtuvo notas que oscilan entre 80 y 97 puntos. Además, $1.47 \%$ de los alumnos no ha realizado el trabajo, por lo que la calificación obtenida fue cero.

En general, los resultados de aprendizaje de los estudiantes obtenidos en todos los trabajos prácticos demuestran que los alumnos son capaces de aplicar con destreza las herramientas de edición y comunicación personal, realizar criteriosamente búsquedas de información, conocer los estándares y gestionar imágenes médicas, analizar y categorizar los datos clínicos, y emplear los softwares de HC para el registro y realizar estadísticas básicas.

Como se observa en la gráfica 2, salvo dos alumnos, el resto ha alcanzado la calificación considerada para la promoción. Con la evaluación de todos los trabajos prácticos se puede concluir que los estudiantes han trabajado de manera adecuada en los grupos de trabajo con intervenciones de los docentes.

Los puntajes obtenidos en el trabajo grupal de integración horizontal con otra asignatura evidencian que los alumnos, gracias a los conocimientos, habilidades y capacidades obtenidas previamente en la materia, han logrado construir nuevos significados y dar un sentido, funcionalidad y aplicación práctica a los temas de IM en una situación real de su profesión; además, en este rubro también se ha observado un compromiso con el trabajo y con el grupo de compañeros. De los datos obtenidos de la evaluación del trabajo (ver gráfica 3), se puede concluir que, en relación con un grupo total de 138 estudiantes, 


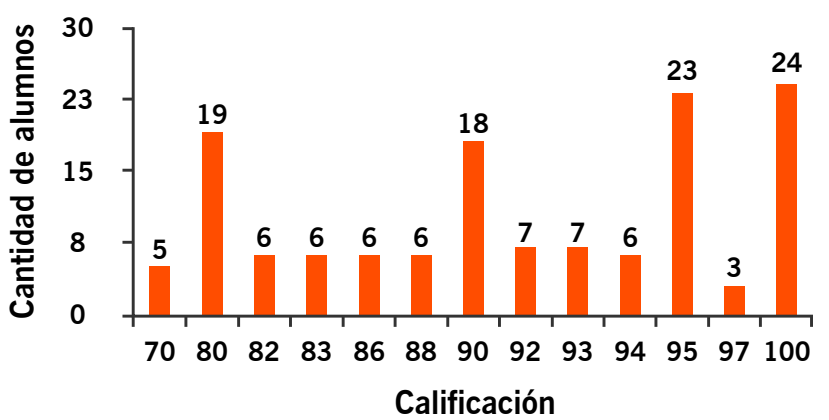

Gráfica 1. Resultados del trabajo práctico Búsqueda de Información Médica.

Fuente: elaboración propia.

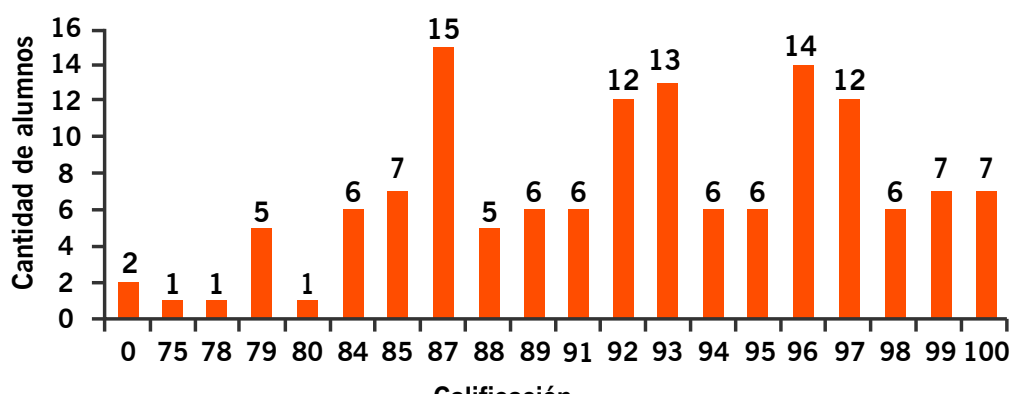

Calificación

Gráfica 2. Promedio de las calificaciones de los trabajos prácticos.

Fuente: elaboración propia.

cinco alumnos no realizaron el trabajo por no cursar la asignatura Salud Pública II. De los 133 alumnos restantes, dos alumnos no presentaron el trabajo, 6\% alcanzó la calificación máxima, mientras que la calificación de $92.4 \%$ oscila entre 80 y 95 puntos.

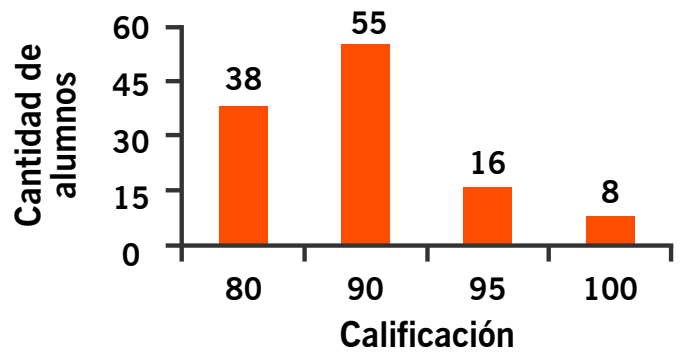

Gráfica 3. Calificaciones del trabajo integrador. Fuente: elaboración propia.
Respecto al trabajo grupal de teoría, en el grupo de 133 alumnos se obtuvo como tendencia la calificación de siete a diez puntos, y la nota nueve se centró como un resultado muy bueno dentro del rango mencionado como aceptado para la promoción (ver gráfica 4).

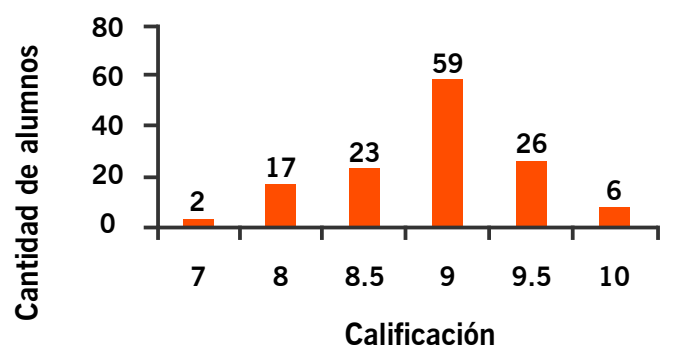

Gráfica 4. Tendencia del resultado de trabajo grupal de teoría. Fuente: elaboración propia. 


\section{Eje 2. Trabajos individuales de valoración práctica y teórica}

En los trabajos individuales se realizó una actividad de valoración sobre los contenidos prácticos de la materia; previo a esto, se informaron las pautas para su realización, momento para el que se habilitó un chat de consultas, cuyo objetivo fue resolver las dudas que se pudieran presentar. De las conversaciones del chat de consultas se desprendió que varios alumnos tuvieron problemas para subir a la plataforma los archivos de la resolución de los ejercicios, ya sea porque se les cerró el cuestionario por excederse en el horario establecido, o por desconocer los mecanismos para subir varios archivos.

De acuerdo con las estadísticas realizadas (ver gráfica 5) se destaca que 72 alumnos (62\%) tienen una nota superior o igual a siete, mientras que $44(38 \%)$ tienen notas menores a siete. A partir de esto, se han alcanzado los siguientes resultados de aprendizajes:
- Analizar y categorizar los datos clínicos, así como emplear los softwares de HC para su registro y procesamiento.

- Analizar el problema, organizar los datos y obtener estadísticas mediante el uso de Excel y EpiInfo.

- Manejar las herramientas para la gestión de imágenes médicas.

Del análisis de los resultados obtenidos, la cátedra decidió realizar varias clases de consultas, de ejercitación adicional y, por último, una evaluación final para los alumnos que no cumplieron con los objetivos de aprendizajes planteados.

En el trabajo de teoría participaron 125 alumnos, de los cuales 83\% (104 alumnos) obtuvo una nota superior o igual a siete, y se registraron 13 ausentes (ver gráfica 6). Se destaca una alta participación de los estudiantes en esta actividad, así como un alto porcentaje de alumnos con notas excelente y muy bueno, lo que permite observar la valoración e importancia que se otorga al proceso de acompañamiento pedagógico.

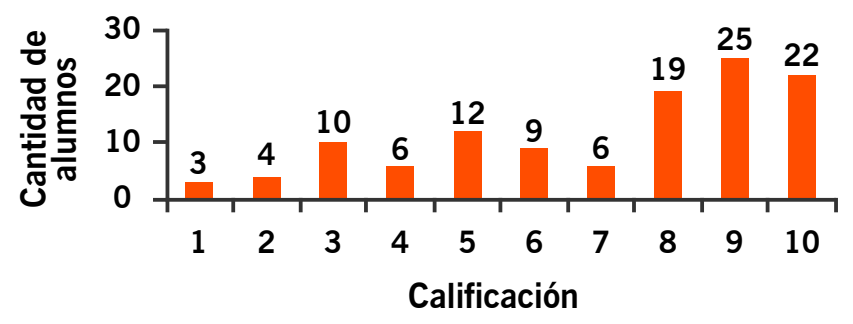

Gráfica 5. Tendencia de la actividad de valoración de la práctica individual. Fuente: elaboración propia.

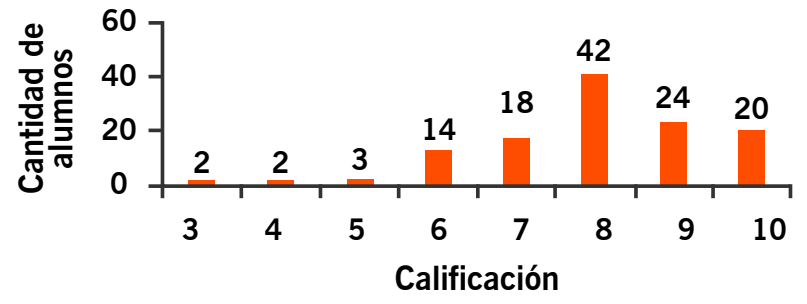

Gráfica 6. Tendencia del cuestionario para autorregulación del aprendizaje teórico. Fuente: elaboración propia. 


\section{Eje 3. Interacciones}

En la interacción alumno-alumno se organizaron 25 grupos de trabajo. Debido al aislamiento preventivo y obligatorio decretado por la pandemia de Covid-19, la comunicación entre ellos se realizó por WhatsApp. Para llevar a cabo el trabajo colaborativo también hicieron uso de Google Drive y del correo electrónico. Por otro lado, la relación de los alumnos con los profesores se efectuó a través de la plataforma Moodle, que permitió obtener estadísticas de esta interacción en relación con los chats y foros. No se obtuvieron estadísticas de las videoconferencias, los correos electrónicos, ni de los mensajes de WhatsApp.

Sobre los chats de consultas se concluye que se han producido 29, la cantidad de estudiantes oscila entre doce y 132 alumnos que participaron e hicieron el seguimiento de este recurso. En cuanto a los foros, se han realizado cinco, y la cantidad de estudiantes que ha visto el recurso oscila entre 19 y 123. En la gráfica 7 se observa el promedio de ocasiones en que un estudiante vio los recursos (chat o foro) de interacción.

Respecto a la interacción del estudiante con el aula virtual, en la gráfica 8 se observa la cantidad de ocasiones en promedio que un estudiante ha visitado el recurso o la actividad del AV.

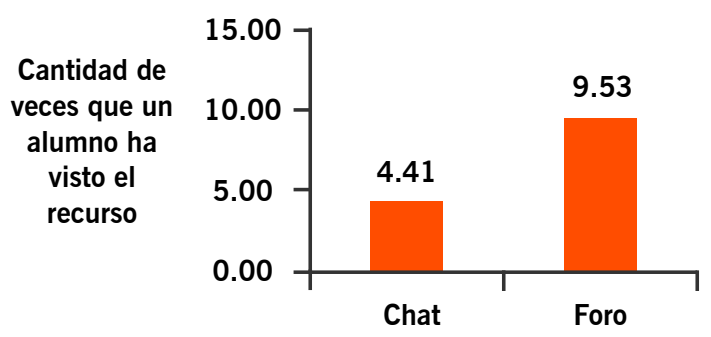

Recurso de interacción

Gráfica 7. Promedio de vistas de recursos de interacción. Fuente: elaboración propia.

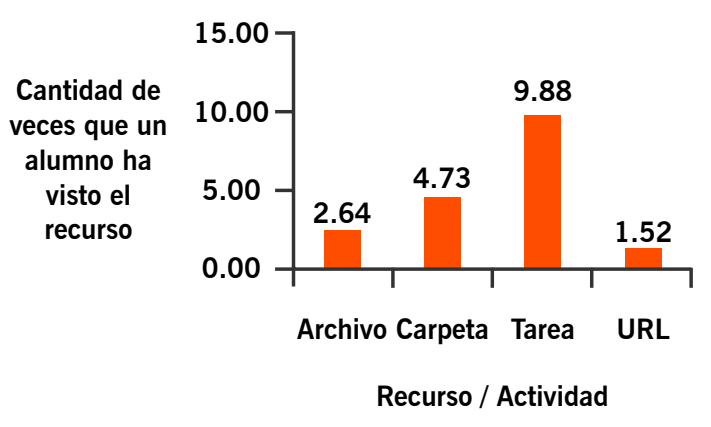

Gráfica 8. Promedio de vistas de un recurso/actividad.

Fuente: elaboración propia.

\section{Eje 4. Intervenciones}

Se determinó que los estudiantes deben observar el proceso y la dinámica de participación del grupo para que se produzca la experimentación, la reflexión y el ajuste de los modos y momentos de participación. Por ello, para alcanzar la participación deseada en las actividades, previamente se definió como requisito en las consignas la participación obligatoria en algunas actividades, de modo que se minimizaran las resistencias respecto de su uso.

\section{CONCLUSIONES}

A partir de los resultados y las evidencias de aprendizaje descritos, el diseño pedagógico propuesto de $\mathrm{AV}$ permitió, en relación con la dimensión informativa, organizar por temas los materiales producidos por los docentes de la cátedra, así como realizar la búsqueda y selección de otros materiales multimediales complementarios. Se considera que la dimensión experimental favoreció las experiencias prácticas de cada unidad temática y, en la dimensión evaluativa, facilitó la incorporación de actividades de valoración teóricas y prácticas para el seguimiento del progreso de los estudiantes, así como de los logros alcanzados acompañados de la retroalimentación de los docentes. El 
diseño pedagógico permitió proporcionar ayudas formativas sobre los contenidos y las actividades prácticas planteadas por la asignatura y la interacción entre los estudiantes, al ser esta la principal aportación sobre la dimensión comunicativa.

En cuanto a la mediación educativa en el AV, permitió organizar y fijar las pautas y los recursos de las estrategias didácticas, lo cual se considera un componente altamente aprovechable para el trabajo docente en los ambientes de enseñanza y de aprendizaje con modalidad virtual, ya que el docente necesita organizar y estructurar su pensamiento con los mecanismos didácticos consecuentes para solventar la clase, de modo que se apliquen las pautas de aprendizaje y trabajo para lograr interés y proactividad entre los estudiantes en el ambiente de enseñanza-aprendizaje.

A partir de la experiencia descrita, se espera incorporar en el ciclo lectivo 2021 el uso de la Guía Didáctica del Estudiante como un mapa y recurso orientativo del $\mathrm{AV}$, ya que por las prontas e inesperadas condiciones sanitarias actuales, no se pudo lograr con la antelación y programación necesaria. En cuanto a las estadísticas analizadas, se estima profundizar el estudio del eje 4, correspondiente a las interacciones, a fin de lograr mayor cohesión y afianzamiento del estudiante.

Finalmente, a partir de los resultados alcanzados en esta experiencia áulica virtual, considerados por esta cátedra como altamente productivos y significativos, en cuanto a la formación académica y como experiencia de innovación pedagógica docente, se podrá solicitar a las respectivas autoridades académicas el permiso para el dictado de la asignatura bajo el modelo mixto de enseñanza-aprendizaje, o blended learning, para el próximo ciclo lectivo 2021.

También, como ampliación de esta experiencia pedagógica, y ya concretada como propuesta de innovación, se desea iniciar una alfabetización digital centrada en el aprendizaje basado en competencias, necesaria para la integración de los estudiantes en la cultura digital y en la sociedad del futuro profesional médico.

\section{REFERENCIAS}

Area, M. (2018). De la enseñanza presencial a la docencia digital. Autobiografía de una historia de vida docente. RED. Revista de Educación a Distancia, (56). Recuperado de: http://www. um.es/ead/red/56/area.pdf

Area, M. y Adell, J. (2009). e-Learning: enseñar y aprender en espacios virtuales, en J. De Pablos (coord.), Tecnología Educativa. La formación del profesorado en la era de Internet. Málaga: Aljibe.

Barberá, E. y Badia, A. (2005). Hacia el aula virtual: actividades de enseñanza y aprendizaje en la red. Revista Iberoamericana de Educación, 36(9). Universidad Oberta de Catalunya. Recuperado de: https://rieoei.org/historico/deloslectores/1064Barbera. PDF

García Aretio, L. (30 de marzo de 2020). LMS. Plataformas virtuales 0 entornos virtuales de aprendizaje. Ventajas y funcionalidades. [Entrada de blog]. Hypotheses. Recuperado de: http://aretio. hypotheses.org/3292

García Fernández, 0. (2014). Estudio de una experiencia: aulas virtuales para apoyar la docencia presencial. Cuadernos de Educación y Desarrollo, Servicios Académicos Intercontinentales, 54. Recuperado de: https://www.eumed.net/rev/atlante/2014/12/aulas-virtuales.html

García-Peñalvo, F. y Seoane Pardo, A. (2015). Una revisión actualizada del concepto de e-Learning. Education in the Knowledge Society, 16(1), 119-144. Recuperado de: https://www.redalyc. org/pdf/5355/535554757008.pdf

Granados-Zúñiga, J. (2019). Relación entre el uso del aula virtual y el rendimiento académico en estudiantes del curso de Bioquímica para Enfermería de la Universidad de Costa Rica. Revista Educación, 43(2). Universidad de Costa Rica. https://doi. org/10.15517/revedu.v43i2.32723

Hoyos Giraldo, F. (2015). Diseño de una plataforma virtual para la enseñanza y aprendizaje de la asignatura Modelos de Información contable prospectiva (trabajo de maestría). Argentina: Facultad de Ciencias Económicas, Universidad de Buenos Aires. Recuperado de: http://bibliotecadigital.econ.uba.ar/download/tpos/1502-0962_HoyosGiraldoFA.pdf

Humanante-Ramos, P.; Fernández-Acevedo, J. y Jiménez, C. (2019). Aulas virtuales en contextos universitarios: percepciones de uso por parte de los estudiantes. Revista ESPACIOS, 40(2). Recuperado de: https://www.revistaespacios.com/a19v40n02/ a19v40n02p03.pdf 
Khan, B. (2005). Managing E-Learning: Design, Delivery, Implementation and Evaluation. Estados Unidos de América: Information Science Publishing.

Monteza Calderón, R. (2016). Uso de las TIC en la Facultad de Humanidades de la USAT (tesis doctoral). Málaga: Universidad de Málaga.

Nivela Cornejo, M. A.; Echeverría Desiderio, S. V. y Espinosa Izquierdo, J. G. (2019). Los proyectos integradores de saberes en el aprendizaje adaptativo. Espirales. Revista multidisciplinaria de investigación científica, 3(25). Recuperado de: https://www.revistaespirales.com/index.php/es/article/view/635/558

Porro, J. (2017). El aula virtual y sus dimensiones: un análisis de la propia práctica. Educación, Formación e Investigación, 3(5).
Recuperado de: http://ppct.caicyt.gov.ar/index.php/efi/article/ view/11100

Rodríguez, L. (2006). Un modelo para la educación en ambientes virtuales. Colombia: Editorial Universidad Pontificia Bolivariana, Escuela de Educación y Pedagogía / Grupo de Investigación Educación en Ambientes Virtuales.

Tecnologías para la Educación (TEDU). (2016). Experimentación de plataformas de aprendizaje. Vicerrectoría de Innovación y Programas en Línea Tecnologías para la Educación 2016. Monterrey, México: Tecnológico de Monterrey. Recuperado de: https://repositorio.tec.mx/bitstream/handle/11285/622386/Re porte + de +Experimentaci\%C3\%B3n+de+Plataformas + de + Aprendizaje + 2016.pdf? sequence $=1$

Este artículo es de acceso abierto. Los usuarios pueden leer, descargar, distribuir, imprimir y enlazar al texto completo, siempre y cuando sea sin fines de lucro y se cite la fuente.

\section{CÓMO CITAR ESTE ARTÍ́CULO:}

Digión, Leda Beatriz y Álvarez, Margarita María. (2021). Experiencia de enseñanza-aprendizaje con aula virtual en el acompañamiento pedagógico debido al Covid-19. Apertura, 13(1), pp. 20-35. http://dx.doi.org/10.32870/Ap.v13n1.1957 